OPEN ACCESS

Edited by:

Vincent Pialoux,

Université Claude Bernard Lyon 1 .

France

Reviewed by:

Theodore Francis Towse,

Grand Valley State University,

United States

Gregory Brown,

University of Nebraska at Kearney,

United States

Emily C. Dunford,

McMaster University, Canada

*Correspondence: Alvaro Reischak-Oliveira alvaro.oliveira@ufrgs.br

Specialty section This article was submitted to

Exercise Physiology,

a section of the journal

Frontiers in Physiology

Received: 25 June 2018 Accepted: 04 June 2019

Published: 21 June 2019

Citation:

Boeno FP, Farinha JB, Ramis TR, Macedo RCO, Rodrigues-Krause J,

Queiroz JN, Lopez P, Pinto RS and Reischak-Oliveira A (2019) Effects of a

Single Session of High-

and Moderate-Intensity Resistance Exercise on Endothelial Function of Middle-Aged Sedentary Men.

Front. Physiol. 10:777.

doi: 10.3389/fphys.2019.00777

\section{Effects of a Single Session of High- and Moderate-Intensity Resistance Exercise on Endothelial Function of Middle-Aged Sedentary Men}

Francesco Pinto Boeno, Juliano Boufleur Farinha, Thiago Rozales Ramis, Rodrigo Cauduro Oliveira Macedo, Josianne Rodrigues-Krause, Jessica do Nascimento Queiroz, Pedro Lopez, Ronei Silveira Pinto and Alvaro Reischak-Oliveira*

Program of Human Movement Sciences, Faculty of Physical Education, Physiotherapy and Dance, Universidade Federal do Rio Grande do Sul, Porto Alegre, Brazil

Regular resistance exercise is associated with metabolic, neuromuscular and cardiovascular adaptations which improve quality of life and health. However, sedentary subjects have shown acute impairments in endothelial function after high-intensity resistance exercise. The aim of this study was to evaluate endothelial function in sedentary middle-aged men after a single session of resistance exercise at different intensities. Eleven sedentary middle-aged men (40.1 \pm 3.9 years; $\left.27.3 \pm 1.4 \mathrm{~kg} / \mathrm{m}^{2}\right)$ underwent three different conditions of assessment: (1) single knee extension exercise at moderate intensity (MI) [4 sets of 12 repetitions at 50\% of one repetition maximum (1RM) for each leg], (2) single knee extension exercise at high intensity $(\mathrm{HI})$ (4 sets of 8 repetitions at $80 \%$ of $1 \mathrm{RM}$ for each leg), (3) resting for the control condition (CON). Flowmediated dilation (FMD) was assessed before, 30 and 60 min after exercise. Plasma concentrations of endothelin-1 (ET-1), nitrites and nitrates $\left(\mathrm{NO}_{\mathrm{x}}\right)$ and thiobarbituric acid reactive substances (TBARS) were measured before, immediately after and 60 min after exercise. Blood pressure (BP) was measured prior to the experimental protocols, and in the following times: immediately following, and 2, 5, 10, 15, 30, and 60 min after exertion. There was a significant improvement in FMD 30 min after MI condition (12.5 \pm 4.10 vs. $17.2 \pm 3.9 \% ; p=0.016)$. $\mathrm{NO}_{x}$ levels were significantly higher immediately after $\mathrm{Ml}(6.8 \pm 3.3$ vs. $12.6 \pm 4.2 \mu \mathrm{M} ; p=0.007)$ and there was a significant increase in the concentration of ET-1 immediately after $\mathrm{HI}(20.02 \pm 2.2$ vs. $25.4 \pm 2.1 \mathrm{pg} / \mathrm{mL} ; p=0.004$ ). However, there was no significant difference for BP (Ml vs. $\mathrm{HI}$ ) and TBARS among the experimental conditions. Resistance exercise performed at moderate intensity improved vasodilatation via increases on NOx levels and FMD in sedentary middle-aged men.

Keywords: resistance exercise, flow mediated dilation, endothelial function, nitric oxide, endothelin-1 


\section{INTRODUCTION}

Physical exercise is related to decreased mortality due to cardiovascular diseases, contributing to the reduction of risk factors and the maintenance of endothelial function (Goto et al., 2003; Inaba et al., 2010; Green et al., 2011; McClean et al., 2015). In this sense, resistance exercise promotes well known significant adaptations to the neuromuscular system, such as increases in the functional reserve of its practitioners. Additionally, in recent years, resistance exercise has been emerging as a prevention and treatment strategy, eliciting positive effects on blood pressure (BP) regulation and insulin sensitivity (Williams et al., 2007).

Despite the aforementioned benefits, the effect of resistance exercise on endothelial function still needs to be clarified. Endothelium plays a crucial role in the regulation of vascular tone through the release of bioactive factors, such as nitric oxide (NO) and endothelin-1 (ET-1) (Shah, 2007; Green et al., 2011). The greater bioactivity of NO leads to antiatherogenic actions, which are triggered by an increase in endotheliumdependent vasodilation. On the other hand, high ET-1 levels are related to arterial hypertension and the progression of atherosclerotic disease (McEniery et al., 2002; Shah, 2007). It has been recently shown that during resistance exercise, the magnitude of ET-1-induced vasoconstriction increases with the aging process (Barrett-O'Keefe et al., 2015). In addition, highintensity resistance exercise elevates ET-1 levels in sedentary individuals (Okamoto et al., 2008). This last effect seems to occur acutely, since resistance exercise chronically reduces ET-1 levels (Maeda et al., 2004).

In this sense, flow-mediated dilation (FMD) can provide a prognostic of endothelial function based in the concept that the direct assessment of the wall vessel response represents the endothelium behavior to environment through the release of vasoactive factors (Jurva et al., 2006; Green et al., 2011; Phillips et al., 2011; Franklin et al., 2014). Acute impairments of FMD, as well as elevations in ET-1 concentrations, may contribute to the lower bioavailability of $\mathrm{NO}$ and the increased incidence of cardiovascular events, especially in individuals with increased risk factors (Green et al., 2011; Thijssen et al., 2011; Dawson et al., 2013; Barrett-O'Keefe et al., 2015). Thus, the role of ET-1 may be a key to understand the endothelial function during resistance exercise. Additionally, the formation of reactive oxygen species (ROS) in response to higher intensities may attenuate endothelium vasodilatory response by decreasing NO bioavailability (Goto et al., 2003).

There is an acute impairment in the FMD of sedentary individuals after maximal resistance exercise, which seems to be related with a transient BP elevation after exercise (Phillips et al., 2011; Franklin et al., 2014). However, the cross-sectional studies aiming to investigate the FMD response to resistance exercise focused on large muscle groups and only used nearmaximal protocols (above $90 \%$ of maximal load) (Jurva et al., 2006; Phillips et al., 2011; Franklin et al., 2014). It suggests that, in this population, higher intensities can lead to this phenomenon. However, studies involving different intensities of exercise, such as moderate resistance exercise, are needed to confirm this hypothesis. Indeed, exercise intensity can directly affect endothelial responses through some adjustments in blood flow pattern, metabolic demand and ROS formation (Goto et al., 2003; Thijssen et al., 2011; McClean et al., 2015).

Therefore, there is a need to understand the endothelial response to different intensities of resistance exercise, as well as the role of the endothelium-derived factor in the vascular tone after resistance exercise in sedentary people (Birk et al., 2012). Thus, the main goal of this study was to evaluate the acute effects of moderate- and high-intensity resistance exercise on FMD and plasma levels of ET-1, nitrites and nitrates (NOx) and thiobarbituric acid reactive substances (TBARS) in sedentary middle-aged individuals.

\section{MATERIALS AND METHODS}

\section{Participants}

Eleven sedentary middle-aged individuals volunteered to participate in this study. The sample size was calculated, and subjects were recruited from the local community through the newspaper advertisement and social media. There were no dropouts during the study. Individuals who performed regular physical exercises, presented cardiovascular diseases or risk factors such as hypertriglyceridemia, hypertension, or diabetes were excluded. Additionally, to participate of the present study the individuals reported at least 6 months without any involvement in exercise programs. This study was approved of the local ethics committee (Permit No. 48788415.1.0000.5347).

\section{Experimental Design}

This randomized cross-over study was composed by 5 days of evaluations separated by at least $72 \mathrm{~h}$. Before every visit to the laboratory, subjects were instructed to perform $12 \mathrm{~h}$ of fasting, not to ingest alcoholic beverages or stimulants, not to perform physical exercises and to maintain the same food pattern $24 \mathrm{~h}$ preceding the tests. Evaluations were performed in the morning (7:00 to $8: 30$ a.m.) to minimize the effects of the circadian cycle on the variables evaluated.

On the first day of evaluations, subjects were submitted to a basal metabolic rate test, a blood test (fasting triglyceridemia and glycemia measurements), and 1RM test familiarization. On the second day of evaluations, the 1RM test was performed to determine the maximum dynamic force of the knee extensor muscles. The experimental protocols were randomly performed by drawing lots, by an independent person, considering the following conditions: high-intensity resistance exercise at $80 \%$ of $1 \mathrm{RM}(\mathrm{HI})$, moderate intensity at $50 \%$ of $1 \mathrm{RM}(\mathrm{MI})$, or resting in the control condition (CON).

Upon arrival at the laboratory, individuals received a standardized meal equivalent to $15 \%$ of basal metabolic rate. 40 min after ingestion, subjects lay in the supine position to perform the following measurements at rest: blood pressure, FMD and blood tests (NOx, ET-1 and TBARS). Right after these resting measurements, the subjects randomly performed one of the three experimental conditions and returned immediately to the supine position. FMD was measured 30 and $60 \mathrm{~min}$ after the end of the exercise protocols. BP was measured before, right 
after and in the following times: 2, 5, 10, 15, 30 and 60 min after physical effort. Blood samples were collected before, immediately after, and $60 \mathrm{~min}$ after each experimental condition.

\section{Experimental Measurements Basal Metabolic Rate}

Basal metabolic rate tests were performed at $20^{\circ} \mathrm{C}-25^{\circ} \mathrm{C}$, with controlled noise and low light. The protocol consisted of 10 min resting in the dorsal decubitus position, followed by 20 min of expired gasses collection. A computerized gas analyser (COSMED, Quark model CPET, Italy) was used to determine the $\mathrm{VO}_{2}$ and $\mathrm{VCO}_{2}$. To calculate basal metabolic rate (kcal/day), we used the equation proposed by Weir: $\left[\left(3.9 \mathrm{X} \mathrm{VO}_{2}\right)+(1.1 \mathrm{X}\right.$ $\mathrm{VCO}_{2}$ )] x 1440 (Weir, 1990).

\section{Maximum Dynamic Strength Test (1RM)}

One repetition maximum (1RM) test was used to quantify the maximum strength values of the knee extensor muscles. Before the test, all subjects were previously familiarized with the equipment and with the procedures of the test (Konnen Gym, Beijing, China). The test was performed for knee extension exercise unilaterally, on both legs, with controlled 2-s metronome pacing for each phase of the exercise. Initially, $5 \mathrm{~min}$ of cycling at $15 \mathrm{~W}$ was performed as warm-up for all the subjects. After warm-up, the subjects were positioned in the equipment and persuaded to start the test. If the individual could perform more than one repetition, a period of 3-min resting was respected between the attempts. Each individual had the maximum of five attempts to complete the test. 1RM was considered as the maximum load in which an individual could perform a repetition with good technique and full range of motion, followed by concentric failure.

\section{Exercise Protocols}

The experimental protocol was designed to mimic a typical resistance exercise set. In this way, the intensities and repetitions were selected based on the general guidelines (Williams et al., 2007). The experimental protocols were performed in a knee extension chair under three conditions: HI, MI, or CON. HI: the exercise was performed unilaterally, consisting of 4 sets of 8 repetitions at $80 \%$ of 1 -RM for each leg. There was 2 min interval between the sets. MI: the exercise was performed under the same general conditions as $\mathrm{HI}$, but the subjects were instructed to perform 4 sets of 12 repetitions at $50 \%$ of $1 \mathrm{RM}$. In both conditions, all the subjects completed all reps without concentric failure. CON: the subjects remained seated in the equipment without making any effort for $15 \mathrm{~min}$. Characteristics of the experimental protocols and participants are shown in Table 1.

\section{Arterial Flow-Mediated Dilatation (FMD)}

The FMD analysis consisted of acquiring images of the brachial artery using a ultrasound system (Nemio XG, Japan) with a $7.5 \mathrm{MHz}$ linear array probe. The position of the ultrasound probe was marked and measured according to the distance from the antecubital crease and all examinations were performed in
TABLE 1 | Sample characterization.

\begin{tabular}{lc}
\hline Variables & Mean \pm SD \\
\hline Age (years) & $40.2 \pm 3.9$ \\
BMl (kg/m²) & $27.3 \pm 1.5$ \\
Triglyceridemia (mg/dL) & $114.9 \pm 25.8$ \\
Glycemia (mg/dL) & $86.7 \pm 7$ \\
Basal metabolic rate (kcal/day) & $1787 \pm 142$ \\
Rest systolic blood pressure (mmHg) & $125.6 \pm 3.7$ \\
Rest diastolic blood pressure (mmHg) & $82.4 \pm 3.2$ \\
Maximum dynamic strength test (1-RM) & \\
Right knee extensors (N) & $627.6 \pm 143.2$ \\
$50 \%$ of 1-RM (N) & $313.8 \pm 74.5$ \\
$80 \%$ of 1-RM (N) & $502.1 \pm 120.6$ \\
Left knee extensors (N) & $604.16 \pm 98.1$ \\
$50 \%$ of 1-RM (N) & $302 \pm 5.2$ \\
$80 \%$ of 1-RM (N) & $498.3 \pm 81.4$ \\
\hline
\end{tabular}

the same position. FMD is an indirect measure of endothelial function and was adapted (Lopes Kruger et al., 2016), following previous guidelines (Thijssen et al., 2011). All evaluations were completed by the same blinded investigator with identical subject/equipment positioning.

The evaluations occurred in an air-conditioned room (21 to $24^{\circ} \mathrm{C}$ ), always in the same period of the day, after $15 \mathrm{~min}$ of rest in the supine position. In the pre-occlusion period, five images were analyzed and the mean values were used as the baseline diameter of the brachial artery. After that, a pressure cuff placed on the forearm of the subjects was inflated to $>240 \mathrm{mmHg}$, maintaining the pressure for $5 \mathrm{~min}$. After $5 \mathrm{~min}$ of occlusion, the cuff was deflated and new images of the brachial artery were obtained with simultaneous electrocardiographic tracing. Three measurements were performed in each experimental condition (i.e., before, 30 and $60 \mathrm{~min}$ after the exercise protocol). All evaluation was recorded on DVD for further analysis in Image-J software.

In order to minimize the influence of the cardiac cycle on the arterial diameter, arterial thickness was determined always occurring in the " $\mathrm{R}$ " wave of the electrocardiogram (Thijssen et al., 2011). As a limitation of the technique, scans of the arterial diameter throughout the post-occlusion period were performed at fixed periods of 60,90 , and 120 seconds after cuff release. The mean dilation value found in each predetermined period was used for analysis. The values of FMD are shown as the percentage of increases in brachial artery diameter related to basal values. The percentage of vasodilation was calculated as follows:

$$
\text { Vasodilation } \%=\frac{[(\mathrm{EH}-\mathrm{EB})]^{*} 100}{\mathrm{~EB}}
$$

EH represents the dilation of brachial artery after reactive hyperaemia and EB the basal thickness of the brachial artery.

\section{Blood Pressure}

Blood pressure was determined by the auscultatory method using a properly calibrated mercury column sphygmomanometer with 
flexible cuff in the appropriate size, and a stethoscope (Littmann, Classic III, United States), as previously described (Franklin et al., 2014). Blood pressure was measured at rest, prior to the experimental protocols and at the end of the protocols at the following times: immediately following, and 2, 5, 10, 15, 30, and 60 min after exertion.

\section{Dietary Control}

Participants were instructed to avoid food rich in nitrites and nitrates $24 \mathrm{~h}$ prior to the sessions of assessment in order to minimize the influence of food intake in plasma levels of $\mathrm{NO}_{\mathrm{x}}$. Additionally, a list of foods rich in nitrites and nitrates was provided for participants to know which foods to avoid. A standardized meal was provided prior to the experimental protocols, which was composed of $62 \%$ carbohydrates, $16 \%$ lipids, and $22 \%$ proteins. The energy content was calculated individually to correspond to $15 \%$ of the basal metabolic rate for 1 day. A sandwich composed of white bread with light cream cheese was offered.

In order to carry out the evaluation of the participants' food consumption, a 24-h food recall (R24) was used. It was filled out as follows: the participant was asked about all food and drink consumed in the last $24 \mathrm{~h}$ before the performance of the three protocols ( $\mathrm{HI}, \mathrm{MI}$, and CON). Data were calculated using the Diet Win Professional Nutrition Software (Brubins CAS, Brazil). Comparisons were made in between the content and quality of food consumed by the participants.

\section{Blood Tests}

Blood samples were taken from the antecubital region by a qualified professional, using sterile and disposable materials. Blood samples were collected into EDTA tubes and centrifuged at $3000 \mathrm{~g}$. Samples were stored at $-80^{\circ} \mathrm{C}$ for further analysis. Analysis of triglyceridemia and glycemia were performed through the colorimetric method using an automated analyser (Cobas C111, Roche, Switzerland). ET-1 levels were determined by enzyme-linked immunosorbent assay (ELISA) using commercial kits for humans (BosterBio, Pleasanton, CA, United States). NOx levels were determined through colorimetric method (Cayman, Ann Arbor, MI, United States). Analysis were performed in duplicate, according to manufacturers' instructions. TBARS concentrations were analyzed as previously described (Ohkawa et al., 1979) and results expressed as $\mu \mathrm{M}$ of $\mathrm{MDA} / \mathrm{L}$. Results were obtained on a microplate reader (Thermo Fisher Scientific, Multiskan Go, Finland) at $532 \mathrm{~nm}$.

\section{Statistical Analysis}

Values are presented as mean \pm standard deviation (SD). Normality of the data was tested by the Shapiro-Wilk test. A twoway analysis of variance (ANOVA) with repeated measures was performed to verify the effect of protocols and moments on the variables. A Bonferroni post hoc was used to locate the differences. Statistical significance was considered for $p \leq 0.05$ and the Statistical Package for Social Sciences (SPSS 20, United States) software was used.

\section{RESULTS}

\section{Participants}

Characteristics of participants and resistance exercise are shown in Table 1.

The standardized meal contained $268 \pm 22 \mathrm{kcal}$. There were no differences in the macronutrient consumption among the three protocols. HI protocol: $1941 \pm 695 \mathrm{kcal}$ (carbohydrates: 54.1 $+10.6 \%$; proteins: $22.2+7.6 \%$; and lipids: $23.6+8.0 \%)$. MI protocol: $2170 \pm 1055 \mathrm{kcal}$ (carbohydrates: $53.6+9.0 \%$; proteins: $21.0+7.5 \%$; and lipids: $25.4+5.0 \%)$. CON protocol: $2076+$ 861 kcal (carbohydrates: $52.5+11.4 \%$; proteins: $19.8+4.6 \%$; and lipids: $27.7+8.6 \%)$.

\section{Arterial Flow-Mediated Vasodilatation (FMD)}

There were no significant variation within-days in the diameter of the brachial artery before protocols $(5.34 \pm 0.6$ vs. $5.46 \pm 07$ vs. $5.30 \pm 0.5 \mathrm{~mm}, p>0.05)$. However, a significant increase of FMD in the brachial artery was found $30 \mathrm{~min}$ after MI condition $(12.5 \pm 4.10$ for $17.2 \pm 3.9 \%, p=0.01)$, returning to baseline levels $60 \mathrm{~min}$ after the protocol. No changes were found after HI or CON conditions. Changes in FMD are shown in Figure 1.

\section{Biochemical Variables}

Concentrations of $\mathrm{NO}_{\mathrm{x}}$ increased at the end of exercise for MI protocol (6.8 \pm 3.3 vs. $12.6 \pm 4.2 \mu \mathrm{M}, p=0.007)$. When comparing the different conditions, levels of $\mathrm{NO}_{\mathrm{x}}$ were significantly elevated after exercise for MI condition compared to $\mathrm{HI}(12.6 \pm 4.2$ vs. $6.59 \pm 3.62 \mu \mathrm{M}, p=0.01)$, and $\mathrm{CON}$ $(12.6 \pm 4.2$ vs. $6.27 \pm 2.22 \mu \mathrm{M}, p=0.001)$. No changes in NOx levels were found after HI or CON conditions. Figure 2 illustrates the concentrations of $\mathrm{NO}_{\mathrm{x}}$ in the different protocols.

Concentrations of ET-1 increased significantly right after $\mathrm{HI}$ $(20.02 \pm 2.2$ vs. $25.4 \pm 2.1 \mathrm{pg} / \mathrm{mL}, p=0.004)$, and decreased $60 \mathrm{~min}$ after exercise $(25.4 \pm 2.1$ vs. $21.5 \pm 4.1 \mathrm{pg} / \mathrm{mL}, p=0.03)$. Moreover, there was a difference at post-exercise for $\mathrm{HI}$ when compared to MI condition $(25.4 \pm 2.1$ vs. $19.8 \pm 5.4 \mathrm{pg} / \mathrm{mL}$,

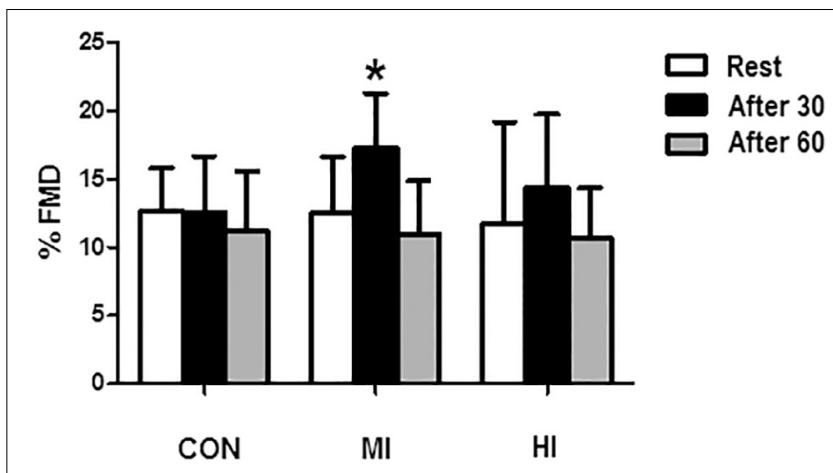

FIGURE 1 | Percentage of brachial artery dilatation in response to different exercise conditions. *Significant difference $(p=0.0016)$ in relation to pre-exercise moment for $\mathrm{Ml}$ condition. CON, control condition; MI, moderate intensity; HI, high intensity. 


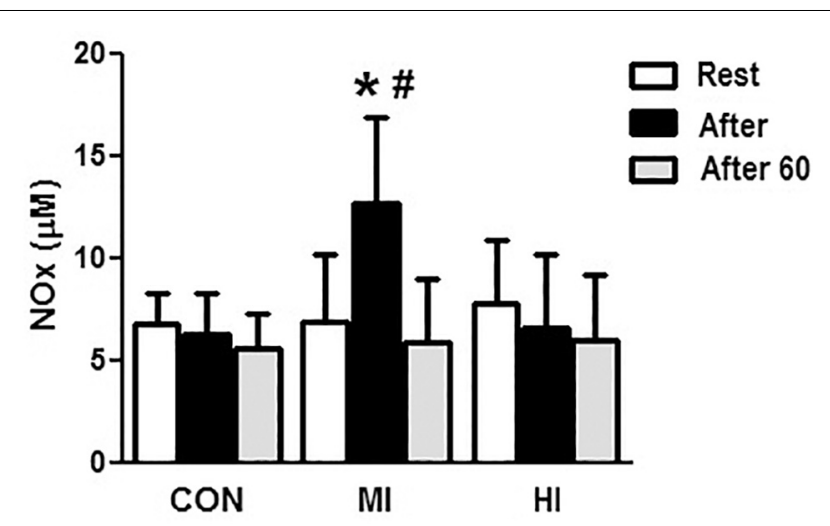

FIGURE 2 | Concentrations of NOx in the plasma in response to different exercise conditions. *Elevated plasma concentrations of NOx at post-exercise moment compared to pre-exercise moment for $\mathrm{Ml}$ protocol ( $p=0.007$ ).

\#Plasma concentrations of NOx significantly elevated at post-exercise moment for $\mathrm{Ml}$ condition compared to $\mathrm{HI}(p=0.015)$ and $\mathrm{CON}(p=0.001)$. $\mathrm{CON}$, control condition; MI, moderate intensity; HI, high intensity.

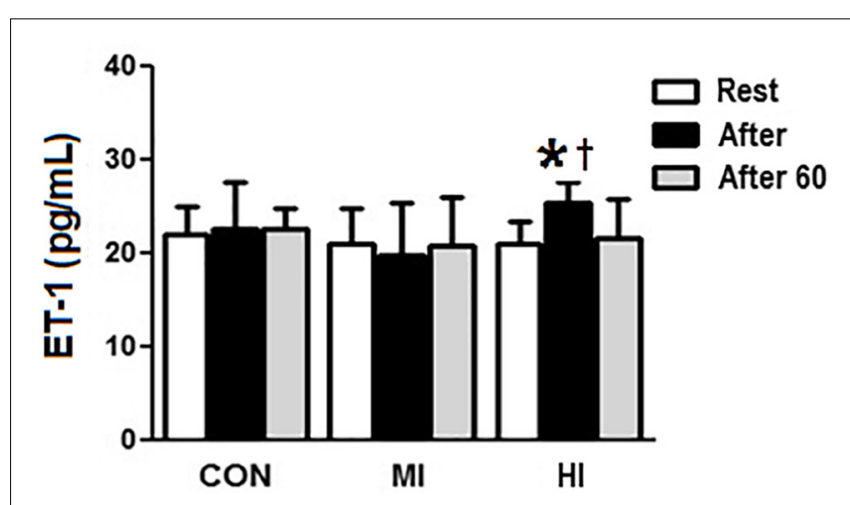

FIGURE 3 | Plasma levels of ET-1 in response to different exercise conditions. *ET-1 levels significantly increased $(p=0.004)$, compared to pre-exercise moment for $\mathrm{HI}$ condition. ${ }^{\dagger} \mathrm{ET}-1$ levels increased significantly at post-exercise moment for $\mathrm{HI}$ condition, compared to $\mathrm{Ml}$ condition $(p=0.002)$. CON, control condition; Ml, moderate intensity; HI, high intensity.

$p=0.002)$, but not when compared to CON $(p>0.05)$. MI and CON conditions did not induce to any significant changes on ET-1 concentrations (Figure 3).

No significant changes were found in plasma levels of TBARS ( $\mu \mathrm{M}$ of $\mathrm{MDA} / \mathrm{L}$ ) for the different exercise conditions, as follows: CON: before: $1.34 \pm 0.96$, after: $1.12 \pm 0.31$, after 60: $1.13 \pm 0.33$. MI: before: $1.38 \pm 0.27$, after: $1.41 \pm 0.24$, after 60 : $1.44 \pm 0.29$. HI: before: $1.26 \pm 0.22$, after: $1.18 \pm 0.76$, after $60: 1.59 \pm 1.21$.

\section{Blood Pressure}

Both MI and $\mathrm{HI}$ conditions resulted in a significant increase in the absolute systolic blood pressure (SBP) in relation to the CON condition $2 \mathrm{~min}$ after exercise (HI: $174 \pm 14 \mathrm{mmHg}$; MI: $158 \pm 26$ mmHg; CON: $124 \pm 8$ mmHg; $p<0.05)$. However, no significant differences were observed between MI and HI conditions. Diastolic blood pressure (DBP) did not change among time and conditions. There was no difference in SBP throughout the following measurements.

\section{DISCUSSION}

The main findings of this study are: (1) moderate resistance exercise significantly increases endothelium-dependent vasodilation $30 \mathrm{~min}$ after the session; (2) moderate resistance exercise increases $\mathrm{NO}_{\mathrm{x}}$ levels immediately after the session; (3) high-intensity resistance exercise increases plasma levels of ET-1 immediately after exercise; (4) it seems there is no impairment to the vascular function (assessed by FMD) in response to MI or HI resistance exercise in sedentary middle-aged men.

Previous studies have shown that sedentary individuals present acute impairment in FMD after exercise (Gonzales et al., 2011; Birk et al., 2012; Dawson et al., 2013; McClean et al., 2015). Resistance exercise, specifically involving large muscle groups and performed at near-maximal intensity, is usually associated with the decrease of FMD, greater sympathetic activity and higher production of ROS (Jurva et al., 2006; Varady et al., 2010; Phillips et al., 2011; Franklin et al., 2014). Increased blood pressure levels afforded by resistance exercise appears to have a negative influence on FMD (Varady et al., 2010; Phillips et al., 2011). Therefore, we carried out the FMD assessments after the normalization of transient BP elevation (30 and $60 \mathrm{~min}$ after exercise) in order to minimize the influence of $\mathrm{BP}$ as a confounder.

It has recently been shown that exercise intensity influences FMD responses, particularly in protocols using hand grip (Atkinson et al., 2015) and cycle ergometer (Birk et al., 2012). This seems to be associated with the muscle size involved and the pattern of exercise-induced blood flow. Evidence has shown that retrograde blood flow leads to a marked decrease in FMD (Chappell et al., 1998; Himburg et al., 2007), while the anterograde flow increases the bioavailability of NO (Green et al., 2005; Tinken et al., 2009). In the present study, FMD was significantly increased only in MI. We speculate that MI induces a predominantly anterograde flow pattern, leading to increased bioavailability of NO. In fact, a significant increase in NOx levels for MI condition was found in our study. Moderateintensity resistance exercise may induce to a laminar pattern of shear stress, stimulating the influx of endothelial calcium $\left(\mathrm{Ca}^{2+}\right)$ (Olesen et al., 1988; Cooke et al., 1991), increasing the activity of endothelial nitric oxide synthase (eNOS) and consequently elevating NO bioavailability (Bussell, 2002). In agreement with our results, Willoughby et al. (2011) demonstrated elevation in NOx levels after exercise in similar conditions (Willoughby et al., 2011). As a matter of fact, elevated levels of NOx after exercise may represent a greater bioactivity of NO, because $85 \%$ of NOx plasma levels seem to be related to $\mathrm{NO}$ formation (Lundberg et al., 2009).

This study also demonstrates that high-intensity resistance exercise can elevate ET-1 levels. However, results regarding the modulation of ET-1 plasma levels in response to resistance exercise are very controversial. Otsuki et al. (2007) demonstrated that strength-trained individuals presented 
higher concentrations of ET-1 at rest when compared to their sedentary pairs (Otsuki et al., 2007). In the study by Okamoto et al. (2008), elevated ET-1 plasma levels were observed after hand grip exercise at $80 \%$ of concentric peak torque (Okamoto et al., 2008). On the other hand, Maeda et al. (2004) reported reduced levels of ET-1 after 8 weeks of strength training at $80 \%$ of 1 RM (Maeda et al., 2004). In fact, elevated ET-1 levels led to notable vasoconstriction and deleterious effects on blood vessels (Masaki, 2004; Shah, 2007; Thijssen et al., 2007). Moreover, McEniery et al. (2002) demonstrated that an infusion of BQ-123, an endothelin-1 type A (ETA) receptor antagonist, increased the vasodilator response to exercise (assessed by plethysmography) when compared to placebo (McEniery et al., 2002). These data suggest an important contribution of the ET-1/ETA vasoconstrictor mechanism not only in rest, but also in response to exercise.

We suggest two possible mechanisms that may have contributed to the increasing of ET-1 concentrations after the HI protocol in our study: (1) redirection of blood flow and (2) increased retrograde blood flow. During resistance exercise, the progressive activation of muscle fibers (quantify of fibers) is higher as the intensity increases (Okamoto et al., 2008). In this sense, higher muscle recruitment in the $\mathrm{HI}$ protocol increases demand of blood flow for active limbs. In this way, ET-1 secretion is elevated in the non-active limbs during exercise due to redirection of blood flow to active recruited muscles (Maeda et al., 1997). Changes in blood flow pattern that are agonistic for endothelin-converting enzyme (ECE) (Green et al., 2005) may have contributed to the elevation in ET-1 levels after HI. Indeed, there is evidence that increased retrograde blood flow following high-intensity exercise is associated with decreased FMD (Birk et al., 2012; Dawson et al., 2013; Atkinson et al., 2015). Thus, a possible retrograde pattern of blood flow may have induced to increases in ET-1 after the HI protocol. However, future studies are needed to confirm that.

It is well stablished that when connected to ETA receptor, ET1 stimulates the opening of L-type $\mathrm{Ca}^{2+}$ channels, which remain open up to 60 min after stimulation (La and Reid, 1995). It is possible that this continuous entry pathway of intracellular $\mathrm{Ca}^{2+}$ limited the FMD response $30 \mathrm{~min}$ after the HI protocol. There is also an inverse relationship between NO bioavailability and ET-1 levels due to the inhibitory action of NO on ECE (Boulanger and Luscher, 1991). In the present study, no relationship was found between $\mathrm{NO}_{\mathrm{x}}$ and ET-1 levels, however, high concentrations of $\mathrm{NO}_{\mathrm{x}}$ and maintenance of ET-1 levels were found in MI. These results are consistent with the aforementioned evidence and with other studies that also demonstrated the inhibitory effect of NO over the ET-1 (Maeda et al., 2001; Otsuki et al., 2007).

Furthermore, impairments in endothelial function are systematically attributed to increases in ROS formation (McClean et al., 2015), mainly due to the formation of peroxynitrite. However, in our study, lipid peroxidation (evaluated by TBARS concentrations) did not change throughout the experimental conditions. These data are in agreement with Zembron-Lacny et al. (2008), which did not find changes in TBARS levels immediately and $24 \mathrm{~h}$ after resistance exercise (Zembron-Lacny et al., 2008). A possible mechanism for the maintenance of TBARS levels may be an acute increase in superoxide dismutase (SOD) and catalase (CAT) activity (Leaf et al., 1997). This may also indicate a maintenance of endothelial function in both experimental conditions, evaluated 30 and 60 min after RE. In addition, no changes were found in ET-1 and $\mathrm{NO}_{\mathrm{x}}$ levels at $60 \mathrm{~min}$ after both conditions, concomitant with the normalization of FMD in this period.

There are some limitations to this study. We recognize that the assessment of oxidative stress by indirect markers only in plasma is a limitation of this study. Measurements of antioxidant enzymes in erythrocytes or leucocytes would be more accurate. Additionally, there was a significant variability regarding the resting FMD values within the $\mathrm{HI}$ group, which could have contributed to the lack of significant difference observed in this condition. Finally, the blood flow analysis, as velocity and pattern, could contribute to add information to the present study.

\section{CONCLUSION}

To the best of our knowledge, this is the first study to analyze endothelial function in response to different intensities of resistance exercise (using large muscle groups) through FMD and vasoactive factors derived from the endothelium. Our results demonstrate that moderate-intensity resistance exercise can increase FMD, along with elevations in plasma NOx concentrations, in middle-aged sedentary men. We also showed that high-intensity resistance exercise ( $80 \%$ of $1 \mathrm{RM}$ ) lead to increases in ET-1 concentrations. However, future studies are needed to investigate the actual contribution of the vasoconstrictor mechanism of ET-1 to FMD. Finally, longitudinal studies purposing resistance exercise training at moderate intensity would help to clarify chronic endothelial adaptations to this type of exercise in sedentary individuals.

\section{ETHICS STATEMENT}

All the experimental procedures of this study were carried out in accordance with the internationally accepted standards for human research and approved by the Ethics Committee of the Universidade Federal do Rio Grande do Sul.

\section{AUTHOR CONTRIBUTIONS}

FB, JF, TR, RCM, JR-K, JNQ, PL, RSP, and AR-O contributed to the design and implementation of the research, analysis of the results, and writing of the manuscript.

\section{FUNDING}

FB, JF, RCM, JR-K, and PL were supported by a fellowship from the CAPES (Coordination for the Improvement of Higher Education Personnel - Brazil). RSP and AR-O are $\mathrm{CNPq}$ (Brazilian National Council for Scientific and Technological Development) Research Productivity Fellows. 


\section{REFERENCES}

Atkinson, C. L., Carter, H. H., Dawson, E. A., Naylor, L. H., Thijssen, D. H., and Green, D. J. (2015). Impact of handgrip exercise intensity on brachial artery flow-mediated dilation. Eur. J. Appl. Physiol. 115, 1705-1713. doi: 10.1007/ s00421-015-3157-1

Barrett-O’Keefe, Z., Ives, S. J., Trinity, J. D., Morgan, G., Rossman, M. J., Donato, A. J., et al. (2015). Endothelin-A-mediated vasoconstriction during exercise with advancing age. J. Gerontol. A Biol. Sci. Med. Sci. 70, 554-565. doi: 10.1093/ gerona/glu065

Birk, G. K., Dawson, E. A., Atkinson, C., Haynes, A., Cable, N. T., Thijssen, D. H., et al. (2012). Brachial artery adaptation to lower limb exercise training: role of shear stress. J. Appl. Physiol. 112, 1653-1658. doi: 10.1152/japplphysiol.01489. 2011

Boulanger, C. M., and Luscher, T. F. (1991). Differential effect of cyclic GMP on the release of endothelin-1 from cultured endothelial cells and intact porcine aorta. J. Cardiovasc. Pharmacol. 17(Suppl. 7), S264-S266.

Bussell, K. (2002). Legless - But still the way forward. Nat. Rev. Drug Discov. 1:331. doi: $10.1038 / \mathrm{nrd} 807$

Chappell, D. C., Varner, S. E., Nerem, R. M., Medford, R. M., and Alexander, R. W. (1998). Oscillatory shear stress stimulates adhesion molecule expression in cultured human endothelium. Circ. Res. 82, 532-539. doi: 10.1161/01.res.82. 5.532

Cooke, J. P., Rossitch, E. Jr., Andon, N. A., Loscalzo, J., and Dzau, V. J. (1991). Flow activates an endothelial potassium channel to release an endogenous nitrovasodilator. J. Clin. Invest. 88, 1663-1671. doi: 10.1172/jc i1 15481

Dawson, E. A., Green, D. J., Cable, N. T., and Thijssen, D. H. (2013). Effects of acute exercise on flow-mediated dilatation in healthy humans. J. Appl. Physiol. 115, 1589-1598. doi: 10.1152/japplphysiol.00450.2013

Franklin, N. C., Ali, M., Goslawski, M., Wang, E., and Phillips, S. A. (2014). Reduced vasodilator function following acute resistance exercise in obese women. Front. Physiol. 5:253. doi: 10.3389/fphys.2014. 00253

Gonzales, J. U., Thompson, B. C., Thistlethwaite, J. R., and Scheuermann, B. W. (2011). Association between exercise hemodynamics and changes in local vascular function following acute exercise. Appl. Physiol. Nutr. Metab. 36, 137-144. doi: 10.1139/h10-097

Goto, C., Higashi, Y., Kimura, M., Noma, K., Hara, K., Nakagawa, K., et al. (2003). Effect of different intensities of exercise on endothelium-dependent vasodilation in humans: role of endothelium-dependent nitric oxide and oxidative stress. Circulation 108, 530-535. doi: 10.1161/01.cir.0000080893. 55729.28

Green, D. J., Bilsborough, W., Naylor, L. H., Reed, C., Wright, J., O’Driscoll, G., et al. (2005). Comparison of forearm blood flow responses to incremental handgrip and cycle ergometer exercise: relative contribution of nitric oxide. J. Physiol. 562(Pt 2), 617-628. doi: 10.1113/jphysiol.2004.07 5929

Green, D. J., Jones, H., Thijssen, D., Cable, N. T., and Atkinson, G. (2011). Flow-mediated dilation and cardiovascular event prediction: does nitric oxide matter? Hypertension 57, 363-369. doi: 10.1161/hypertensionaha.110.16 7015

Himburg, H. A., Dowd, S. E., and Friedman, M. H. (2007). Frequency-dependent response of the vascular endothelium to pulsatile shear stress. Am. J. Physiol. Heart Circ. Physiol. 293, H645-H653. doi: 10.1152/ajpheart.01087.2006

Inaba, Y., Chen, J. A., and Bergmann, S. R. (2010). Prediction of future cardiovascular outcomes by flow-mediated vasodilatation of brachial artery: a meta-analysis. Int. J. Cardiovasc. Imaging 26, 631-640. doi: 10.1007/s10554010-9616- 1

Jurva, J. W., Phillips, S. A., Syed, A. Q., Syed, A. Y., Pitt, S., Weaver, A., et al. (2006). The effect of exertional hypertension evoked by weight lifting on vascular endothelial function. J. Am. Coll. Cardiol. 48, 588-589. doi: 10.1016/j.jacc.2006. 05.004

La, M., and Reid, J. J. (1995). Endothelin-1 and the regulation of vascular tone. Clin. Exp. Pharmacol. Physiol. 22, 315-323.

Leaf, D. A., Kleinman, M. T., Hamilton, M., and Barstow, T. J. (1997). The effect of exercise intensity on lipid peroxidation. Med. Sci. Sports Exerc. 29, 1036-1039. doi: 10.1097/00005768-199708000-00008
Lopes Kruger, R., Costa Teixeira, B., Boufleur Farinha, J., Cauduro Oliveira Macedo, R., Pinto Boeno, F., Rech, A., et al. (2016). Effect of exercise intensity on postprandial lipemia, markers of oxidative stress, and endothelial function after a high-fat meal. Appl. Physiol. Nutr. Metab. 41, 1278-1284. doi: 10.1139/ apnm-2016-0262

Lundberg, J. O., Gladwin, M. T., Ahluwalia, A., Benjamin, N., Bryan, N. S., Butler, A., et al. (2009). Nitrate and nitrite in biology, nutrition and therapeutics. Nat. Chem. Biol. 5, 865-869. doi: 10.1038/nchembio.260

Maeda, S., Miyauchi, T., Iemitsu, M., Sugawara, J., Nagata, Y., and Goto, K. (2004). Resistance exercise training reduces plasma endothelin-1 concentration in healthy young humans. J. Cardiovasc. Pharmacol. 44(Suppl. 1), S443-S446.

Maeda, S., Miyauchi, T., Kakiyama, T., Sugawara, J., Iemitsu, M., IrukayamaTomobe, Y., et al. (2001). Effects of exercise training of 8 weeks and detraining on plasma levels of endothelium-derived factors, endothelin-1 and nitric oxide, in healthy young humans. Life Sci. 69, 1005-1016. doi: 10.1016/s0024-3205(01) 01192-4

Maeda, S., Miyauchi, T., Sakane, M., Saito, M., Maki, S., Goto, K., et al. (1997). Does endothelin-1 participate in the exercise-induced changes of blood flow distribution of muscles in humans? J. Appl. Physiol. 82, 1107-1111. doi: 10.1152/ jappl.1997.82.4.1107

Masaki, T. (2004). Historical review: endothelin. Trends Pharmacol. Sci. 25, 219224. doi: 10.1016/j.tips.2004.02.008

McClean, C., Harris, R. A., Brown, M., Brown, J. C., and Davison, G. W. (2015). Effects of exercise intensity on postexercise endothelial function and oxidative stress. Oxid. Med. Cell Longev. 2015:723679. doi: 10.1155/2015/723679

McEniery, C. M., Wilkinson, I. B., Jenkins, D. G., and Webb, D. J. (2002). Endogenous endothelin-1 limits exercise-induced vasodilation in hypertensive humans. Hypertension 40, 202-206. doi: 10.1161/01.hyp.0000024218.04872.f3

Ohkawa, H., Ohishi, N., and Yagi, K. (1979). Assay for lipid peroxides in animal tissues by thiobarbituric acid reaction. Anal. Biochem. 95, 351-358. doi: 10. 1016/0003-2697(79)90738-3

Okamoto, T., Masuhara, M., and Ikuta, K. (2008). Relationship between plasma endothelin-1 concentration and cardiovascular responses during high-intensity eccentric and concentric exercise. Clin. Physiol. Funct. Imaging 28, 43-48. doi: 10.1111/j.1475-097X.2007.00775.x

Olesen, S. P., Clapham, D. E., and Davies, P. F. (1988). Haemodynamic shear stress activates a K+ current in vascular endothelial cells. Nature 331, 168-170. doi: $10.1038 / 331168 \mathrm{a} 0$

Otsuki, T., Maeda, S., Iemitsu, M., Saito, Y., Tanimura, Y., Ajisaka, R., et al. (2007). Vascular endothelium-derived factors and arterial stiffness in strength- and endurance-trained men. Am. J. Physiol. Heart Circ. Physiol. 292, H786-H791. doi: 10.1152/ajpheart.00678.2006

Phillips, S. A., Das, E., Wang, J., Pritchard, K., and Gutterman, D. D. (2011). Resistance and aerobic exercise protects against acute endothelial impairment induced by a single exposure to hypertension during exertion. J. Appl. Physiol. 110, 1013-1020. doi: 10.1152/japplphysiol.00438.2010

Shah, R. (2007). Endothelins in health and disease. Eur. J. Intern. Med. 18, 272-282. doi: 10.1016/j.ejim.2007.04.002

Thijssen, D. H., Black, M. A., Pyke, K. E., Padilla, J., Atkinson, G., Harris, R. A., et al. (2011). Assessment of flow-mediated dilation in humans: a methodological and physiological guideline. Am. J. Physiol. Heart Circ. Physiol. 300, H2-H12. doi: 10.1152/ajpheart.00471.2010

Thijssen, D. H., Rongen, G. A., van Dijk, A., Smits, P., and Hopman, M. T. (2007). Enhanced endothelin-1-mediated leg vascular tone in healthy older subjects. J. Appl. Physiol. 103, 852-857. doi: 10.1152/japplphysiol.00357.2007

Tinken, T. M., Thijssen, D. H., Hopkins, N., Black, M. A., Dawson, E. A., Minson, C. T., et al. (2009). Impact of shear rate modulation on vascular function in humans. Hypertension 54, 278-285. doi: 10.1161/hypertensionaha.109.13 4361

Varady, K. A., Bhutani, S., Church, E. C., and Phillips, S. A. (2010). Adipokine responses to acute resistance exercise in trained and untrained men. Med. Sci. Sports Exerc. 42, 456-462. doi: 10.1249/MSS.0b013e3181ba6dd3

Weir, J. B. (1990). New methods for calculating metabolic rate with special reference to protein metabolism. 1949. Nutrition 6, 213-221.

Williams, M. A., Haskell, W. L., Ades, P. A., Amsterdam, E. A., Bittner, V., Franklin, B. A., et al. (2007). Resistance exercise in individuals with and without cardiovascular disease: 2007 update: a scientific statement from the american heart association council on clinical cardiology and council on nutrition, 
physical activity, and metabolism. Circulation 116, 572-584. doi: 10.1161/ circulationaha.107.185214

Willoughby, D. S., Boucher, T., Reid, J., Skelton, G., and Clark, M. (2011). Effects of 7 days of arginine-alpha-ketoglutarate supplementation on blood flow, plasma L-arginine, nitric oxide metabolites, and asymmetric dimethyl arginine after resistance exercise. Int. J. Sport Nutr. Exerc. Metab. 21, 291-299. doi: 10.1123/ ijsnem.21.4.291

Zembron-Lacny, A., Ostapiuk, J., Slowinska-Lisowska, M., Witkowski, K., and Szyszka, K. (2008). Pro-antioxidant ratio in healthy men exposed to muscledamaging resistance exercise. J. Physiol. Biochem. 64, 27-35. doi: 10.1007/ bf03168232
Conflict of Interest Statement: The authors declare that the research was conducted in the absence of any commercial or financial relationships that could be construed as a potential conflict of interest.

Copyright (c) 2019 Boeno, Farinha, Ramis, Macedo, Rodrigues-Krause, do Nascimento Queiroz, Lopez, Pinto and Reischak-Oliveira. This is an open-access article distributed under the terms of the Creative Commons Attribution License (CC BY). The use, distribution or reproduction in other forums is permitted, provided the original author(s) and the copyright owner(s) are credited and that the original publication in this journal is cited, in accordance with accepted academic practice. No use, distribution or reproduction is permitted which does not comply with these terms. 